\title{
Public distress as a moral consideration in after- birth abortion
}

\author{
P. Biegler \\ Centre for Human Bioethics, Monash University \\ paul.biegler@monash.edu
}

In a recent paper, Giubilini and Minerva argue that in cases where in utero abortion is currently condoned, 'after-birth abortion', or infanticide, ought also to be permitted. ${ }^{1}$ For example, a third trimester abortion might be defended on the basis of foetal genetic abnormality, or through appeal to unacceptable parental suffering should the child live. On the authors' formulation, infanticide in neonates of the same corrected age, in otherwise identical circumstances, ought also to be defended. The argument appeals to the parity principle. If there exist no relevant moral differences between two cases, and the first is ethically defensible, then the second case should also be deemed permissible. ${ }^{2}$ The paper has, unsurprisingly, provoked widespread public criticism, and even revulsion. In what follows, I argue that the distress evoked by these kinds of arguments is a moral consideration that ought to play a more prominent role in the debate.

Liberal bioethics typically casts the permissibility of abortion as a balance between the interests of the foetus and those of the mother. To have an interest is to have a good that can be set back or furthered. Individuals with interests can, correspondingly, be harmed or benefitted. The earliest foetal interest is commonly held to be avoidance of pain. Pain perception is possible from around 16 weeks, suggesting the foetus has an interest in averting pain from that gestational age. ${ }^{3}$ Inflicting pain at or after this stage comprises a harm to

1 Alberto Giubilini and Francesca Minerva, 'After-birth abortion: why should the baby live?' Journal of Medical Ethics, published online 23 February 2012. Available from: http://jme.bmj.com/content/early/2012/03/01/medethics-2011-100411.full.

2 Søren Holm, 'If you have said A, you must also say B: is this always true?', Cambridge Quarterly of Healthcare Ethics 13 (2), 2004, 179-84.

3 Marc Van de Velde et al., 'Fetal pain perception and pain management', Seminars in Fetal and Neonatal Medicine 11 (4), 2006, 232-6. 
the foetus. As the physiological capacities of the foetus increase so too do its range of interests. For example, as auditory processing develops exposure to loud unpleasant noise might be construed as a harm.

Extending this account, it has been argued that an interest in life requires an organism to conceive both the notion of continued existence and itself as the subject of that state. ${ }^{4}$ A prominent view is that the cognitive capacity for such conceptualisation is absent until toddlerhood. The conclusion is that, prior to this age there is no interest in life and, because rights presuppose interests, no corresponding right to life. A corollary is that if life is terminated at or prior to this age, no interest is thwarted, no right transgressed, and no harm perpetrated. Establishing that the foetus lacks a right to life supports the claim that the maternal harm of continued pregnancy outweighs any putative harm to the terminated foetus.

Frequently missing in this debate is discussion of how community interests might inform the morality of abortion and, by extension, its 'postpartum' counterpart. Respect for personal autonomy is often adduced as reason to circumscribe abortion to a mother's decision about her body, over which she is sovereign. Infanticide, however, shifts the moral landscape, because the mother's body is no longer the venue for debate. Ex utero, the child becomes a bona fide citizen, increasingly subject to the influence of societal norms. One such norm stresses the warrant for vulnerable beings, including children, to be afforded community protection. Adults who, for example, see a child wandering into harm's way will commonly intervene, even if the child is not their own. This norm is formalised in the various government arrangements that attempt to monitor children's welfare, and intervene should it be critically compromised.

These social norms have a strong evolutionary pedigree. In-group altruism makes a forceful claim to be innate. Primates who view con-specifics in pain exhibit distress in the form of immobility and anorexia. ${ }^{5}$ Moreover, the intensity of distress varies directly with the closeness of the relationship with

4 Michael Tooley, 'Abortion and infanticide', Philosophy and Public Affairs 2 (1), 1972, 37-65.

5 Mark D. Hauser, Wild minds: what animals really think, New York: Henry Holt and Company, 2000, 223. 
the victim. On one view, this type of emotional response is adaptive in that it reinforces practices that assist survival of a group. Protection by the group enhances, in turn, survival of individuals. Adult revulsion at infanticide shares, in all likelihood, evolutionary origins. Concern for the safety of young, and distress should they encounter danger, probably evolved because they are critical to offspring survival. This view is also consistent with an evolutionary theory of emotion. ${ }^{6}$ Negative emotions index occurrences of disvalue, and motivate efforts to abate those contingencies. Negative emotional responses to infanticide may persist as echoes of an outcome boding ill for the prospects of a social group.

The question for moral theory is whether abhorrence of 'after-birth abortion' is a reliable contemporary indicator of disvalue. Infanticide may be more widely viewed as permissible in severe disability or illness with terrible prospects of survival. Repugnance seems misplaced in these scenarios. Indeed, when the facts of such cases are disseminated, (legal) decisions to with hold life prolonging therapy are often viewed with relief rather than hostility. Yet, where infanticide is advocated for comparatively minor disability or illness, or indeed when the child is healthy, negative emotions have far stronger claim to warrant. That warrant may no longer reflect evolutionary reasons for altruism - stretched global resources suggest we should curtail rather than expand the population. People do, nonetheless, derive a tangible good from the knowledge their society protects the vulnerable from harm. It is for example, a source of solace that laws exist to shield children from abuse, enable access to vaccinations, make the walk to school safer, and ensure teachers are appropriately trained. It gives comfort to know that ambulances have benchmark response times and that paediatric emergency departments are equipped to deal with life threatening illness or misadventure. In short, there is utility in the knowledge that children are protected, a utility reinforced by the distressing emotions that accompany transgressions in this domain.

Working backwards, this account provides partial explanation for the ire provoked by late term abortions. It is no coincidence that abortion triggers

6 Ray J. Dolan, Emotion, cognition, and behavior', Science 298 (5596), 2002, 1191-4. 
stronger emotions as the foetus becomes more recognisably human. A wealth of evidence shows that humans react more intensely, and become more motivated when data concerning human suffering is 'anthropomorphised'? Thus, statistics detailing a multitude of childhood deaths are often ignored while the image of a single suffering infant can provoke mass grief. Perhaps this kind of response in abortion can also claim evolutionary origins. Being recognisably human has no small correlation with survival prospects: up to $60 \%$ of first trimester pregnancies miscarry, and reaching the third trimester significantly increases foetal viability.

This framework suggests one way in which negative emotional responses to late term and 'after birth' abortion may indicate negative utility. That disvalue pertains to the interest our society has in being confident that the weak and vulnerable will be afforded protection. Arguments of the form put forward by Giubilini and Minerva are broadly consequentialist in appealing to the overall utility of actions. As a signal of disutility, public distress warrants much greater consideration in debates on the permissibility of these practices.

7 Tehila Kogut and Ilana Ritov, 'The 'identified victim' effect: an identified group, or just a single individual?' Journal of Behavioral Decision Making 18, 2005, 157-167. 